\title{
Correction to: Mental Health in China: Stigma, Family Obligations, and the Potential of Peer Support
}

\author{
Shelly $\mathrm{Yu}^{1}$ - Sarah D. Kowitt ${ }^{1}$ Edwin B. Fisher ${ }^{1}$. Gongying $\mathrm{Li}^{2,3,4}$
}

Published online: 4 January 2018

○) Springer Science+Business Media, LLC, part of Springer Nature 2018

\section{Correction to: Community Mental Health Journal https://doi.org/10.1007/s10597-017-0182-z}

The original version of this article unfortunately contained a mistake in "Funding" section. The funding information for $\mathrm{Dr}$. $\mathrm{Li}$ is missing in the original publication. The corrected Funding section is given below:

Funding Ms. Kowitt, Ms. Yu and Dr. Fisher were supported by Peers for Progress at the Gillings School of Global Public
Health at the University of North Carolina. At the time the research was conducted, it was a program of the American Academy of Family Physicians Foundation, supported by the Eli Lilly and Company Foundation and the Bristol-Myers Squibb Foundation. Dr. Li was supported by The National Natural Science Fund of China (81571334).

Sarah D. Kowitt

kowitt@email.unc.edu

$\triangle$ Gongying Li

ligongying2005@126.com

1 Peers for Progress and Department of Health Behavior, Gillings School of Global Public Health, University of North Carolina at Chapel Hill, Rosenau Hall, CB \#7440, Chapel Hill, NC 27599-7440, USA

2 School of Mental Health and Institute of Behavioral Medicine, Jining Medical University, No. 16 Hehua Rd. Taibaihu New District, Jining 272067, Shandong, China

3 School of Mental Health and Institute of Behavioral Medicine, Jining Medical University, No. 16 Hehua Rd. Taibaihu New District, Jining 272013, Shandong, China

4 The Second Affiliated Hospital, Jining Medical University, North Dai Zhuang, Rencheng District, Jining 272051, Shandong, China 